\title{
Overcoming barriers to hepatitis B immunisation by a dedicated hepatitis $B$ immunisation service
}

\author{
V F Larcher, J Bourne, C Aitken, D Jeffries, D Hodes
}

\begin{abstract}
Aims-To determine the effectiveness of a selective hospital based hepatitis B immunisation programme and the barriers to be overcome in obtaining a successful outcome.

Methods-Retrospective case note review of 265 infants born over a five year period to hepatitis B carrier mothers at a university affiliated hospital in Hackney, London.

Results-A total of 242 infants (91\%) were fully vaccinated; 217 (82\%) had serology; 31 required booster doses. Percentages failing to reach second, third vaccinations, and serology on schedule rose exponentially $(7 \%, 18 \%, 33 \%$ respectively). Mobility was high (25\%) and significantly affected outcome. A total of $95 \%$ Hackney resident babies were fully vaccinated compared with $78 \%$ non-residents. Uptake of routine immunisations was higher in Hackney residents than non-residents and greater in those who were eligible for hepatitis $B$ vaccine. Name changes occurred in $35 \%$. Translation requirements were high $(85 \%$ for Turkish, Vietnamese, and Asian families). Requirements for specific postnatal counselling of mothers and hepatology referral fell significantly during the course of the study. Only seven of 22 babies born in 1995 in Tower Hamlets compared with 53 of 58 Hackney babies received a full vaccination course in non-hospital based primary care.
\end{abstract}

Conclusion-In inner city areas with high prevalence of hepatitis $B$ carriage, mobility, and diverse ethnicity, a dedicated centralised immunisation service can be highly effective, provided that adequate support services (translation, counselling, and parental referral) are available. (Arch Dis Child 2001;84:114-119)

Keywords: hepatitis B; immunisation; inner city; barriers to immunisation

In the UK selective immunisation of all babies born to infected mothers is the recommended method of preventing vertical transmission of hepatitis B and the concomitant risk of chronic liver disease. ${ }^{12}$ The NHS Executive required health authorities to implement universal antenatal screening and appropriate immunisation of all babies born to infected mothers by April $2000 .^{3}$ The Executive neither stipulated where or by whom the immunisation programmes were to be delivered nor what levels of uptake should be achieved. Published data suggest that levels of uptake in UK community and primary care based hepatitis $\mathrm{B}$ immunisation programmes ${ }^{45}$ are generally lower than in centrally organised programmes in the Nether- lands. ${ }^{6}$ In the UK many hepatitis B carriers live in inner cities where uptake of routine immunisations, for example, DPT (diphtheria, pertussis, and tetanus) and MMR (measles, mumps, and rubella) tends to be poor. ${ }^{78}$

Both Hackney and Tower Hamlets are inner city areas which form part of the East London and City Health Authority (ELCHA). They have broadly similar demographic characteristics and similar high indices of social deprivation. The London Borough of Hackney has a total population of 200000 and an under 14 population of 48 000. In 1992-93 babies born to ethnic minorities comprised $48 \%$ of live births; $31 \%$ were black African or Caribbean.

A hospital based hepatitis B immunisation programme was established in Hackney in 1991 following the introduction of universal antenatal screening. Here we evaluate the effectiveness of this programme over a five year period and analyse the barriers to be overcome in achieving a successful outcome.

\section{Materials and methods}

Hepatitis B infected mothers were identified at booking by universal antenatal screening at the Homerton Hospital, Hackney, London. All positive women were counselled as to their status, its implication for themselves, their partners, and their families, and the need for immunisation of the baby. One of us (VL) reviewed details of their status from the virology department and was responsible for coordinating the immunisation programme, including development of an in house vaccination protocol and the training of labour ward staff in its use.

Infants of hepatitis B surface antigen (HbsAg) positive, hepatitis B antibody positive mothers (low risk carriers) were scheduled to receive recombinant vaccine $(10 \mu \mathrm{g})$ at birth, 1 , and 6 months. Infants born to high risk mothers (hepatitis B e antigen positive, or $\mathrm{HbsAg}$ positive and hepatitis $\mathrm{B}$ e antibody negative, or acute hepatitis in pregnancy) received in addition 200 IU of hepatitis B specific immunoglobulin at birth. Serological responses to vaccination were tested at one year to establish the effectiveness of the immune response. Those having anti-HBs titres greater than 100 IU/1 were regarded as immune, those with titres of 10-100 were recalled for a booster dose and retesting six weeks later, and those with titres less than 10 had a repeat course of vaccine.

In order to facilitate attendance, the immunisation clinic was held in the hospital at the same time as the neonatal follow up clinic, with nursing and reception staff from the special care baby unit (SCBU). Weekly clinics were held, but an opportunistic policy for vaccine administration was followed for late comers and those who inadvertently attended the SCBU. 


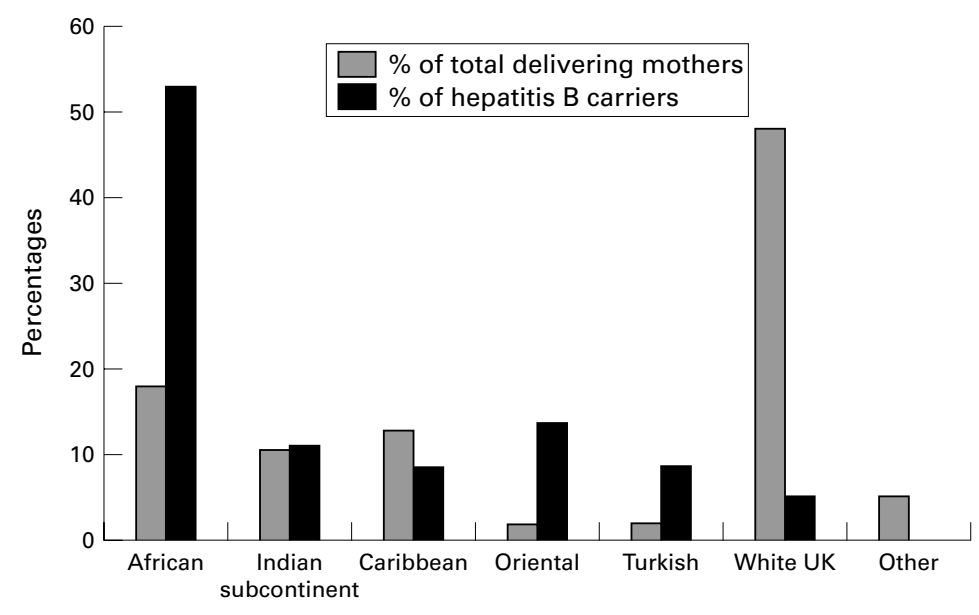

Figure 1 Ethnicity of mothers. studied compared with white mothers, this effect being most notable in African, Oriental, and Turkish groups.

\section{VACCINE UPTAKE}

During the study period there were 18046 live births at the Homerton Hospital: 265 infants $(1.45 \%)$ were born to HBsAg positive mothers of whom $46(17 \%)$ were identified as high risk. Of the eligible infants, $242(91 \%)$ received three doses of vaccine and 217 (82\%) had serological responses measured. Three patients required revaccination, of whom two responded fully. Booster doses were required for 31 infants; 18 attended for post booster serology and all had satisfactory serological responses.

Two children born to high risk mothers became HBsAg positive. One had received no vaccine whatever; the mother had delivered elsewhere and had not disclosed her hepatitis status. The other child failed to complete her course of vaccine but was subsequently tested and identified as HBsAg positive at another hospital. general practitioner, health visitor, and community paediatrician and given the next available appointment. If they failed to attend a second time, hospital based liaison health visitors contacted the family's health visitor and attempted to contact the family themselves to reinforce the need for attendance.

Data from babies born during 1992-96 inclusive were recorded. These included maternal ethnicity, timing of vaccine administration, serology outcomes, and failures to attend.

As part of an attempt to evaluate the effectiveness of the programme in overcoming barriers to immunisation, the following additional data were recorded.

- In 1992-93, need for translation facilities, postnatal education/counselling of families, and hepatology referral were documented.

- In 1995 we evaluated the mobility of the population by recording changes of names and addresses. Details of changes of name and address were obtained from personal health records and cross checked with the regional interactive child health computer system (RICHS). We compared the uptake of routine immunisation given at 6 months (third DPT) and 1 year (MMR). We also recorded the need for postnatal counselling and hepatology referral as a comparison with 1992-93 to provide some measure of the effectiveness of an antenatal counselling service.

Comparative data for hepatitis $\mathrm{B}$ vaccine uptake were obtained for 1995 from a neighbouring London district (Tower Hamlets) where there is a primary care based vaccination programme, but where the number of babies born to hepatitis B carriers is less than in Hackney.

\section{Results}

ETHNICITY

Figure 1 shows the ethnic distribution of hepatitis B carrier mothers in comparison with the ethnic distribution of the total population of delivering mothers. Hepatitis B carriers are seen to be over represented in all ethnic groups
BABIES LOST TO FOLLOW UP

Twenty three (9\%) infants were lost to follow up and presumed to have failed to complete a full course of vaccine. One had no vaccine (see above), seven had only one dose, and 15 two doses. A significant number of those lost to follow up lived outside Hackney ( $\mathrm{n}=14$, $\left.\chi^{2}=17.4, p<0.001\right)$. A significant proportion of babies were African $\left(n=18, \chi^{2}=6.6\right.$, $\mathrm{p}<0.02) ; 12$ were living in Africa and three outside London, but in the UK.

\section{ADHERENCE TO IMMUNISATION SCHEDULES}

Details of the time of administration of the first vaccine dose were available for 184 infants; 180 (98\%) received this within 48 hours of birth and 164 within 24 hours. Only four infants had their first dose after seven days, of whom two subsequently tested negative for hepatitis B markers and were successfully immunised. Delays in attending for the 1 and 6 month doses of vaccine were cumulative, occurring in 16 and 40 respectively of the 217 infants whose serological responses were tested. Late attendance had no effect on serological responses. Testing serology was delayed in 72 infants $(33 \%)$.

EFFECT OF RESIDENCE ON VACCINE UPTAKE A high percentage of infants (66 of $265,25 \%$ ) moved out of Hackney. Of those who remained, a significantly higher proportion (190 of 199) received three doses of vaccine compared with non-residents (52 of 66). $\left(\chi^{2}=17.41, \mathrm{p}<0.001\right)$. A similar trend was observed for serological responses. Data for non-residents were obtained from those who continued to attend for hepatitis vaccination or via health service records and RICHS.

ATTENDANCE FOR ROUTINE IMMUNISATION IN 1995 The uptake of routine immunisation $(\mathrm{DPT} \times 3$ and MMR) in those eligible for hepatitis B vaccine was compared with that in the non-eligible, in both Hackney residents (table 
Table 1 Hepatitis B immunisation versus routine immunisation in Hackney resident babies, 1995

\begin{tabular}{lll}
\hline & Hepatitis B eligible & $\begin{array}{l}\text { Non-hepatitis B } \\
\text { eligible }\end{array}$ \\
\hline Total cases & $40(100)$ & $3218(100)$ \\
Hepatitis B $\times 3$ & $39(97)$ & N/A \\
DPT $\times 3$ & $40(100)$ & $2928(91)$ \\
MMR & $39(97)$ & $2764(86)$ \\
\hline
\end{tabular}

Figures in brackets are percentages. N/A, not applicable.

Table 2 Hepatitis B immunisation versus routine immunisation in non-Hackney resident babies, 1995

\begin{tabular}{lll}
\hline & Hepatitis B eligible & $\begin{array}{l}\text { Non-hepatitis B } \\
\text { eligible }\end{array}$ \\
\hline Total cases & $18(100)$ & $37(100)$ \\
Hepatitis B $\times 3$ & $14(78)$ & N/A \\
DPT $\times 3$ & $9(50)$ & $22(59)$ \\
MMR & $7(39)$ & $18(49)$ \\
\hline
\end{tabular}

Figures in brackets are percentages. N/A, not applicable.

Table 3 Requirement for postnatal maternal counselling and hepatology referral

\begin{tabular}{llrl}
\hline & $1992-93$ & 1995 & $\chi^{2}, p$ value \\
\hline Total number of cases & 98 & 58 & - \\
Number requiring counselling & 47 & 9 & $15.9, \mathrm{p}<0.001$ \\
Number requiring referral & 25 & 2 & $12.8, \mathrm{p}<0.001$ \\
\hline
\end{tabular}

1) and non-residents (table 2). Uptake of routine immunisation was high in the hepatitis B vaccine eligible group of Hackney residents (table 1), but low in the non-resident babies. The hepatitis B eligible babies had a higher proportion of recorded mobility than others.

OTHER POTENTIAL BARRIERS TO HEPATITIS B IMMUNISATION

Name changes

Babies were initially registered in their mother's surname or second name. In 1995, 35\% of infants were recorded as having name changes in the first year of life. The predominant populations affected were West African, Oriental, and Bengali babies; there were too few babies born to white hepatitis B carrying mothers to make meaningful comparisons.

Failure to understand the importance of vaccination Requirement for translation-In 1992-93 translators or health advocacy services were required for 23 of 27 (85\%) families of Indian, Bengali, Turkish, or Vietnamese origin. Overall almost $30 \%$ of hepatitis B carrying mothers required translation services.

Requirements for postnatal counselling/referral to hepatology-Comparison was made for the need for postnatal counselling and hepatology referral between the two periods of 1992-93 and 1995. The numbers of patients requiring postnatal counselling and hepatology referral fell significantly (see table 3 ).

COMPARISON WITH A NEIGHBOURING INNER CITY AREA- 1995

A total of 53 of $58(91 \%)$ Hackney infants compared with seven of $22(32 \%)$ Tower Hamlets infants completed their course of vaccines $\left(\chi^{2}=27, p<0.01\right)$. None were lost to follow up, although one was living abroad. In contrast, nine of $22(41 \%)$ Tower Hamlets infants had been lost to follow up.

\section{Key messages}

- Even in inner city areas over $90 \%$ of infants can receive full hepatitis B immunisation

- Mobility (changes of address) and poor understanding impede uptake

- Effective antenatal screening and counselling, availability of translation, and hepatology referral services may all improve uptake

- Despite problems of accessibility and cost, a centralised service is technically efficient at immunising babies against hepatitis B in inner city areas with high prevalence rates

Costs

We have calculated the total cost (1999 information) of the immunisation programme including vaccines, staff (medical, nursing, secretarial), follow up serology, postage, etc to be circa $£ 32000$. This represents a cost of immunisation of approximately $£ 138$ per successfully immunised baby and $£ 126$ if all babies are taken into account. Staff costs were $59 \%$ of the total cost.

These figures must be set against the long term cost of managing hepatitis B carriers produced by failure of immunisation.

\section{Discussion}

The effective delivery of a selective immunisation programme for babies depends on the accurate identification of all high risk pregnancies by universal screening. Efficient, timely communication of positive results, including mothers' e antigen/antibody status is essential. Those responsible for coordinating the programme and those who will administer the first dose of vaccine must be informed. Counselling of mothers is likely to improve their engagement in the immunisation programme. In our cases this was achieved by one of us receiving virology reports, and checking that labour ward staff were conversant with the vaccination policy. Active checking of maternal status was important because both late bookings and delivery elsewhere (acknowledged problems in inner city areas $^{9}$ ) are potential obstacles to engagement in an immunisation programme. A high proportion of our infants received their first dose of vaccine with or without immunoglobulins within 48 hours.

Although delivery of the first dose of vaccine is important in minimising risk of vertical transmission, effective protection of the infant depends on the completion of a course of vaccine. The percentage of infants receiving a full course of vaccine consistently exceeded $90 \%$, with over $80 \%$ having serological confirmation of their responses.

These coverage figures exceed those previously published for pre-1998 vaccination schedules in the UK, where $21-67 \%$ of babies from Manchester and the Midlands respectively were documented as having received three doses of vaccine. ${ }^{45}$ In an earlier study for the Public Health Laboratory, 89 of 102 babies 
from an original cohort of 155 received three doses of vaccine. ${ }^{7}$ In the Netherlands, where there is a centrally organised service with different vaccination schedules, uptake of three doses of vaccine rose from $80 \%$ to $94 \%$ between 1989 and $1991 .{ }^{6}$ Although an increasing number of our babies failed to receive their second or third doses on schedule, this did not seem to be a barrier to effective immunisation as judged by final outcome. Overall the percentage who did receive vaccine on schedule was higher than those in other series. ${ }^{4} 6$ Nonetheless $9 \%$ were lost to follow up before completing their course and some of these have probably become hepatitis B carriers.

This coverage was achieved in an inner city area where routine immunisation uptake, for example, DPT and MMR is traditionally poor $^{78}$; uptake of third DPT was $91 \%$ and MMR $85 \%$ in Hackney in 1995. In contrast to other studies, uptake of hepatitis B immunisation was greater overall than that of routine immunisations. ${ }^{4}{ }^{6}$ Uptake of DPT 3 and MMR were both higher in Hackney resident babies eligible for hepatitis B than those who were not, suggesting that mothers of these babies may have had an increased awareness of the overall value of immunisation. In non-residents uptake of hepatitis B was much higher than that of routine immunisations. One possible explanation of the latter is that the organisation and delivery of the hepatitis $\mathrm{B}$ immunisation programme overcame the barriers to effective immunisation, which exist in inner cities. ${ }^{7}$ These include a high prevalence of ethnic minority families, one parent families, young mothers, and mobility.

Ethnic minority families were disproportionately represented in this study, emphasing the relatively high prevalence of hepatitis B carriage. ${ }^{10}$ The main barrier to immunisation in ethnic minority families is said to be poor understanding of English. ${ }^{7}$ Immunisation requires valid consent, ${ }^{2}$ which must be adequately informed. Adequate understanding of information may be impossible without translation facilities, which were required by approximately $30 \%$ of all our families. Even if parental command of English is good, difficulties in comprehending the nature and significance of maternal hepatitis B carriage and its consequences for families may still prevent effective immunisation. This may be countered by pretest discussion and post-test counselling and referral of parents and families to hepatology services. ${ }^{3}$ The increasing effectiveness of antenatal counselling is one explanation for the decline for the need for postnatal counselling and hepatology referrals between 1992-93 and 1995 in the absence of a significant proportion of hepatitis B carriers having second or subsequent children in 1995.

Although a significant proportion of our babies were born to young, single mothers, this did not seem of itself to be a barrier to immunisation. Of greater practical difficulty in contacting non-attenders were name changes. We attempted to overcome this by amending the parent held records, cross checking with hospital and other records, liaising with family health visitors and clarifying the infants' name(s) via translators. We also encouraged parents to take responsibility for their own child's immunisation programme by active education, support, and demonstration of the importance of the parent held records.

The mobility of families is a further barrier to immunisation. Hepatitis B carriers were disproportionately more mobile when compared with other Hackney families. Changes of address did have an adverse effect on uptake in that a significant proportion of those lost to follow up lived out of borough and many lived abroad. Nevertheless we did achieve a high coverage in non-residents, which may have been an effect of enhanced parental understanding of the importance of completing a course of vaccine.

Hospital based immunisation services may have potential disadvantages in that they are of limited availability and accessibility. We overcame this by offering an open door opportunistic vaccination policy for those who did not attend clinic at specified times, using centralised and parent held records to register such vaccinations. A further means of overcoming such barriers is the use of community or primary care based services which would also have advantages in terms of costs. However, acknowledged difficulties exist in meeting targets for routine immunisation in inner city areas. ${ }^{7811}$ Experience with hepatitis B immunisation in our neighbouring district and elsewhere suggests that compliance is still suboptimal. ${ }^{12}$ The reason for this may be difficulties in central coordination of such a service, especially in areas where the prevalence of hepatitis B is low. Further, some ethnic minority families may have greater familiarity with or preference for a hospital based mode of delivery of health care.

Hospital based services are likely to be more expensive than those which are community based and our costs were relatively high. They do have to be set against the medical and social costs of poor coverage, which include those of chronic hepatitis B carriage and chronic liver disease. Assuming a similar coverage rate to our neighbouring health district in the absence of a hospital based service, we might expect our 265 infants to have produced approximately 50 new hepatitis B carriers, of whom $25 \%$ would develop chronic liver disease.

These results do not indicate where infants should attend for their hepatitis B immunisation. Rather they suggest that barriers to immunisation in an inner city area may be overcome by the provision of a centralised, dedicated service, which has definable features. These include antenatal pretest discussion, post-test counselling, and hepatology referral; effective communication between virology departments, central coordinators, and those responsible for an initial immunisation; tenacity and persistence in tracing defaulters; skilled translation services; and referral of other family members for testing, counselling, and treatment. Our model meets the requirements of the NHS Executive's recommendations. ${ }^{3}$ 
1 Department of Health: Welsh Office, Scottish Office, Home and Health Department, DHSS (Northern Ireland) and Health Department, DHSS (Northern Ireland). Hepatitis B. In: Immunisation

2 Boxall EH, Zukerman A, eds. Preventing hepatitis $B$ in the new born, children and adolescent. London: Royal College of Physicians, 1996.

3 Department of Health. Screening of Pregnant Women for Hepatitis B and Immunisation of Babies at Risk. HSC 1998 12T. London: Stationery Office, 1998.

4 Wallis DE, Boxall EH. Immunisation of infants at risk of perinatal transmission of hepatitis B. Retrospective audit of vaccine uptake. BM7 1999;318:1112-13.

5 Smith CP, Parle M, Morris DJ. Implementation of government recommendation for immunising infants at risk of hepatitis B. BMF 1994;309:1339.

6 Grosheide PM, Klokman-Houvweling JM, Conyn V, Spaendonck MAE, and the National Hepatitis B Steering Group. Programme for preventing hepatitis B infection Group. Programme for preventing hepatitis B infection through screening of pregnant women and immunisation of infants of affected mother

7 Polakoff S, Vandervelde EM. Immunisation of neonates at high risk of hepatitis B in England and Wales: national surhigh risk of hepatitis B in England
veillance. $B M \mathcal{F} 1988 ; 297: 249-53$.

$8 \mathrm{Li}$ J, Taylor B. Factors affecting uptake of measles, mumps and rubella immunisation. BMF 1993;307:68-71.

9 Deinhardt F, Zuckermann AJ. Immunisation against hepatitis $\mathrm{B}$; report on a WHO meeting on viral hepatitis in Europe. f Med Virol 1985;17:209-17.

10 Peckham C, Bedford H, Senturia Y, Ades A. The Peckham Report-national immunisation study; factors influencing immunisation uptake in childhood. London: Action Research for the Crippled Child, 1989

$11 \mathrm{Li} \mathrm{J}$, Taylor B. Comparison of immunisation rates in general practice and child health clinics. BMf 1991;303:1035-8.

12 Wheeley SM, Boxall EH, Tarlow MJ, et al. Hepatitis B vaccine in the prevention of perinatally transmitted hepatitis B infection; final report in a West Midlands pilot study. $\mathcal{F}$ Med Virol 1990;30:113-16.

\section{Commentary}

The UK is one of the few western European countries which have chosen not to comply with the WHO recommendations for universal hepatitis $\mathrm{B}$ vaccination. The WHO recommended that countries such as the UK, with a low prevalence of carriage, should introduce universal adolescent and/or infant vaccination by $1997 .{ }^{1}$ The UK position therefore needs defending.

Most recognised new hepatitis B infections in the UK occur in young adults ${ }^{2}$; very few infections are acquired in childhood. If we estimate the number of UK infections that lead to chronic carriage, however, adult exposures are the major cause but perinatal infections form a substantial minority. ${ }^{2}$ The most immediate priorities for prevention, therefore, are to target those at greatest risk by improving uptake in those exposed by sexual activity or intravenous drug use, and in infants born to carrier mothers. ${ }^{3}$

Universal adolescent vaccination has potential to impact on adult infections in the medium term, but would have limited impact on perinatal infection as most pregnant carriers will have been infected during childhood in countries of high prevalence. ${ }^{4}$ If universal infant vaccination was adopted, for example using combination infant vaccines, it would take many years to have an impact on the peak incidence in young adults and, unless an additional dose of hepatitis $\mathrm{B}$ vaccine was given at birth, would not prevent perinatal infections. Even with a universal adolescent or infant vaccination policy, therefore, it is still necessary to identify babies born to carrier mothers so that they can be given the additional protection of vaccine at birth and, if the mother is high risk (HBeAg positive and anti-HBe negative), hepatitis B immunoglobulin. ${ }^{5}$ Universal antenatal screening, as recommended since April
2000 , is essential so that the risk status of all babies is known by the date of delivery. ${ }^{6}$

One argument for switching to universal vaccination in infancy is that it would be easier to achieve high vaccine coverage in infants at highest risk. This argument was based on a number of audits which suggested that coverage for selective hepatitis B vaccination was low. ${ }^{7-9}$ Larcher et al show that achieving high hepatitis $\mathrm{B}$ vaccination coverage is possibleeven in an inner city district with a highly mobile and ethnically diverse population. This and studies from overseas have shown that success depends on having a well focused, and actively managed programme with intensive attention to follow up. ${ }^{10-12}$

The prevalence of carriage in the UK antenatal population is very low-probably between $0.1 \%$ and $0.3 \%{ }^{4}{ }^{13}{ }^{14}$ As there are about 640000 births per year in England and Wales, we estimate that around 2000 babies born each year are at risk of perinatal infection. As neonatal vaccination is $90 \%$ effective at preventing a risk of up to $25 \%$ that the child will become a hepatitis B carrier, only four children need to be vaccinated to prevent one carrier. We argue that resources should be targeted at selective infant vaccination and improving follow up of these 2000 infants at greatest risk (including testing for HBsAg). This is likely to have a more rapid impact on perinatal infections and subsequent population $\mathrm{HBsAg}$ carriage than vaccination of 638000 infants who are at no risk at birth and at very low risk during childhood. To justify the UK policy of universal antenatal screening and selective vaccination, however, requires surveillance systems to be in place. Paediatricians have an important role in ensuring that the UK can show that implementation of selective infant vaccination is effective.

Specialist Registrar in Public Health Medicine

DAVID SLOAN

East London and The City Health Authority

MARY RAMSAY

Consultant Epidemiologist

PHLS Communicable Disease Surveillance Centre

61 Colindale Avenue

London NW9 5EQ

mramsay@phls.nhs.uk

1 WHO. Health 21 - health for all in the 21st century. European Health for All series No.6. World Health Organisation, Regional Office for Europe, 1999

2 Ramsay M, Gay N, Balogun K, Collins M. Control of hepatitis B in the United Kingdom. Vaccine 1998;16(suppl): S52-5.

3 Mortimer PP, Miller E. Commentary: antenatal screening and targeting should be sufficient in some countries. BMF 1997;314:1036.

4 Boxall E, Skidmore S, Evans C, Nightingale S. The prevalence of hepatitis B and C in an antenatal population prevalence of hepatitis B and $C$ in an antenatal population

5 Andre FE, Zuckerman AJ. Review: protective efficacy of Andre FE, Zuckerman AJ. Review: protective efficacy of
hepatitis B vaccines in neonates. $\mathcal{F}$ Med Virol 1994;44:14451 .

6 NHS Executive. Screening of pregnant women for hepatitis $B$ and immunisation of babies at risk. HSC 1998/127. London: Department of Health, 1998

7 Smith CP, Parle M, Morris DJ. Implementation of government recommendations for immunising infants at risk of hepatitis B. BMF 1994;309:1339.

8 Wallis DE, Boxall EH. Immunisation of infants at risk of perinatal transmission of hepatitis B: retrospective audit of vaccine uptake. BMF 1999;318:1112-13.

9 Dunn J, Shukla R, Neal K. Survey of neonatal hepatitis B vaccination in Leicestershire. Commun Dis Public Health 1999;2:218-19.

10 Okun NB, Bryce Larke RP, Waters JR, et al. Success of a program of routine prenatal screening for hepatitis B surface antigen: the first 2 years. CMAf 1990;143:131721. 
11 Marion SA, Pastore MT, Pi DW, Mathias RG. Long-term follow-up of hepatitis B vaccine in infants of carrier mothers. Am 7 Epidemiol 1994;140:734-46.

12 Roome AJ, Rak M, Hadler JL. Prevention of perinatal hepatitis B through enhanced case management-Connecticut, 1994-95, and the United States, 1994. MMWR Morb Mortal Wkly Rep 1996;45:584-7.

13 Brook MG, Lever AML, Kelly D, et al. Antenatal screening for hepatitis B is medically and economically effective in the prevention of vertical transmission: three years experience in a London hospital. $Q f M$ 1989;264:313-17.

14 Banatvala JE, Chrystie IL, Palmer SJ, Kenney A. Retrospective study of HIV, hepatitis B and HTLV-1 infection at a London antenatal clinic. Lancet 1990;335:859-60.

\section{Commentary}

Larcher et al show that, in certain instances, a selective approach to hepatitis B virus (HBV) vaccination can work. Accordingly, the UK Department of Health's recent instruction ${ }^{1}$ that all pregnant women should be offered and recommended a hepatitis B test is welcome; this new guidance stemmed from the recognition that large proportions of certain high risk groups were not being vaccinated despite long standing directives which indicated that they should be.

The World Health Organisation, in an attempt to eradicate HBV globally, asked all governments to implement universal HBV vaccination of children in their countries by $1997 .^{2}$ The nearly 100 countries in which all children are now immunised against HBV includes most of Europe and North America. ${ }^{3}$ In the UK, however, a compelling case for the introduction of universal vaccination has been hindered by the equivocality of the results of cost effectiveness analyses ${ }^{45}$; these incorporated relatively scanty data on $\mathrm{HbsAg}$ carriage rates which are lower than those observed in countries which have adopted universal immunisation. Accordingly, the universal approach is still being resisted in favour of a targeted intervention. This "evidence based" stance would be applauded if it were not for the following factors.

In the context of a targeted programme, the vaccination of all those belonging to certain high risk groups, in particular injecting drug users, is problematic. Although the guidance to immunise injectors was launched in $1988,{ }^{5}$ rates of vaccine uptake among this group are either unknown or low. ${ }^{6}$ In Glasgow, a city with the highest prevalence of injecting in the UK and an extensive network of harm reduction agencies, community wide surveys of injectors who had been injecting for, on average, six years, were conducted in 1993/94 and 1999; $81 \%(833 / 1024)$ and $79 \%(351 / 444)$, respectively, had never received a single dose of HBV vaccine (unpublished data, SCIEH). Occasional islands of high uptake have been observed in England, ${ }^{7}$ and the Department of Health has recently pumped considerable resources into improving vaccination rates. ${ }^{8}$ Optimal rates can only be achieved if dedicated funding for vaccination is sufficient and sustained, and if drug treatment/needle and syringe exchange centres are numerous, accessible, and user friendly enough to attract high proportions of injecting populations. ${ }^{7}$ Even if favourable conditions existed throughout the UK, it would not be possible to offer vaccine to those who had recently commenced their injecting careers and had not yet gravitated to such agencies. Past and present experience informs us that vaccinating, for example, over $80 \%$ of UK injectors who had started to inject in the previous two years is unachievable.

In the late 1990s the incidence of acute HBV infection among injecting drug users, UK wide, increased dramatically. ${ }^{9}{ }^{10}$ This observation highlights the pitfall of using HBV data that apply to current and past (injecting) behaviours to make a decision about the implementation of universal vaccination, the impact of which-in terms of the number of hepatitis B infections prevented-depends entirely on the future behaviour of the population; if, for example, the universal vaccination of UK adolescents had been implemented in 1990, a large proportion of the UK's recent HBV infections would have been avoided.

Perhaps the most unconvincing aspect of the current selective policy is that it is not particularly selective. To not merit vaccination one has to eschew-throughout one's lifetimeinjecting drug use, having multiple sexual partners, being imprisoned, working in occupations in which one might come into contact with "sharps", and spending lengthy periods in parts of the world where infection is endemic. ${ }^{11}$ Since millions of persons already qualify for vaccination, the case for continuing with a selective policy appears untenable.

If the above reasons for abandoning the present policy and embracing universal immunisation were still considered insufficient, the premise that the fight to rid this infection globally can only be won if every country, regardless of its current prevalence, is signed up to vaccinate its entire childhood population, should be decisive.

DAVID GOLDBERG

Department of Public Health

University of Glasgow and

Scottish Centre for Infection and Environmental Health Clifton House

Clifton Place

Glasgow G3 7LN, UK

david.goldberg@scieh.csa.scot.nhs.uk

CLAIRE BRAMLEY

Scottish Centre for Infection and Environmental Health

1 Department of Health. Screening of pregnant women for hepatitis $B$ and immunisation of babies at risk. 1998. HSC 1998/12T. London: Department of Health, 1998.

2 World Health Organisation. Expanded programme on immunisation global advisory group. Whly Epidemiol Rec 1992;67:11-16.

3 Van Damme P, Kane M, Meheus A, on behalf of the Viral Hepatitis Prevention Board. Integration of hepatitis B vaccination into national immunisation programmes. $B M \mathcal{F}$ 1997;314:1033-7.

4 Goldberg D, McMenamin J. The United Kingdom's hepatiis B immunisation strategy-where now? Commun Dis Public Health 1998;1:79-83.

5 Edmunds WJ. Universal or selective immunisation against hepatitis B virus in the United Kingdom? A review of recent cost-effectiveness studies. Commun Dis Public Health 1998;1:221-8.

6 Lamagni TL, Davison KL, Hope VD, et al. Poor hepatitis B vaccine coverage in injecting drug users: England, 1995, and 1996. Commun Dis Public Health 1999;2:174-7.

7 Heptonstall J. Strategies to ensure delivery of hepatitis B Heptonstall J. Strategies to ensure delivery of hepatitis B
vaccine to injecting drug users. Commun Dis Public Health vaccine to injecti

8 Department of Health NHS Executive. Drug misuse special allocation: 1999/2000. Funding and guidance on the modernisation fund element. Health Service Circular 1999/036.

9 CDSC. Acute hepatitis B in injecting drug users is increasing as initiative to improve vaccine coverage begins. Commun Dis Rep CDR Wkly 1999;9:183-6.

10 Scottish Executive Health Department. Health in Scotland. ISBN 1842684027,1999

11 UK Health Departments. Immunisation against infectious disease. London: HMSO, 1996. 